VOL. 76 (2007) [353-368]

\title{
A SEQUENTIAL QUADRATIC PROGRAMMING ALGORITHM FOR NONLINEAR MINIMAX PROBLEMS
}

\author{
QING-JIE Hu AND JU-ZHOU Hu
}

\begin{abstract}
In this paper, an active set sequential quadratic programming algorithm with nonmonotone line search for nonlinear minmax problems is presented. At each iteration of the proposed algorithm, a main search direction is obtained by solving a reduced quadratic program which always has a solution. In order to avoid the Maratos effect, a correction direction is yielded by solving the reduced system of linear equations. Under mild conditions without the strict complementarity, the global and superlinear convergence can be achieved. Finally, some preliminary numerical experiments are reported.
\end{abstract}

\section{INTRODUCTION}

It is well known that many engineering design problems can be expressed in a minimax form as follows

(P) $\min \left\{F(x) \mid x \in R^{n}\right\}$,

where $F(x)=\max \left\{f_{j}(x), j \in I\right\}$ with $I=\{1,2, \ldots, m\}$, which enables the designer to minimise the maximum violation of design specifications, (see $[11,16,18,20]$ ). Since the objective function $F(x)$ contains the max operator, it is continuous but nondifferentiable even when the $f_{i}(x), i=1, \ldots, m$, are all differentiable. In particular, at points where the $\max$ is attained in two or more functions $f_{i}(x), F(x)$ is nondifferentiable. So, the classical methods for smooth optimisation problems may fail to reach an optimum if they are applied directly to the nonlinear minmax problem.

Many of the schemes that have been proposed for solving minimax problems are based on an equivalent translation of the original problem (1.1) as follows

$$
\left(\mathrm{P}^{\prime}\right) \min \left\{y \mid(x, y) \in R^{n+1}: f_{j}(x) \leqslant y, j \in I\right\}
$$

but there are drawbacks to use the form $\left(\mathrm{P}^{\prime}\right)$ directly to obtain a solution of (1.1) since some properties of the primal problem may be thrown away (see [15]).

Received 7th March, 2007

The authors thank the anonymous referees for their valuable comments and suggestions on the original version of this paper.

Copyright Clearance Centre, Inc. Serial-fee code: 0004-9727/07 \$A2.00+0.00. 
Obviously, the Karush-Kuhn-Tucker conditions of (1.2) can be stated as follows

$$
\begin{aligned}
& \left(\begin{array}{l}
0 \\
1
\end{array}\right)+\sum_{j \in I} \lambda_{j}\left(\begin{array}{c}
\nabla f_{j}(x) \\
-1
\end{array}\right)=0, \\
& \lambda_{j} \geqslant 0, \quad f_{j}(x)-y \leqslant 0, \quad \lambda_{j}\left(f_{j}(x)-y\right)=0, \quad j \in I,
\end{aligned}
$$

and these relationships are equivalent to

$$
\sum_{j \in I} \lambda_{j} \nabla f_{j}(x)=0, \quad \sum_{j \in I} \lambda_{j}=1 ; \quad \lambda_{j}\left(f_{j}(x)-F(x)\right)=0, \quad \lambda_{j} \geqslant 0, j \in I .
$$

So, a point $x \in R^{n}$ is called as stationary point of (P) ([5]) if there exists a vector $\lambda=\left(\lambda_{j}, j \in I\right)$ such that (1.3) holds, where $\lambda$ is said to be multiplier vector.

Since the sequential quadratic programming method has satisfactory convergence, it is one of the most effective algorithms for solving nonlinearly constrained optimisation problems and it is studied widely and deeply, (see $[10,12,8,7,17,4]$ ). So several authors have extended the popular sequential quadratic programming scheme to the minimax problems (see $[1,9,14,13,21,22]$ ). Global convergence is usually ensured by means of a suitable line search. Based on the equivalent relationship between the KarushKuhn-Tucker point of $\left(\mathrm{P}^{\prime}\right)$ and the stationary point of $(\mathrm{P})$, many algorithms focus on finding the stationary point of $(\mathrm{P})$, namely solving (1.3). Zhou and Tits proposed an algorithm ([23]): the search direction is generated by solving two quadratic programs, and avoiding the Maratos effect by means of nonmonotone line search. Unfortunately, it obtains only two-step superlinear convergence and needs the strict complementarity (namely the multiplier $\lambda_{j}>0$ for $j$ satisfying $f_{j}(x)=F(x)$ ) which is rather difficult for testing. Recently, some sequential quadratic programming algorithms have been proposed to overcome the shortcoming of the two-step superlinear convergence and obtain one-step superlinear convergence, such as [21], but their assumptions are rather strong: (1) the iteration sequence $\left\{x^{k}\right\}$ generated by the algorithm is assumed to satisfy $\lim _{k \rightarrow \infty} x^{k}=x^{*}$; that is, the algorithm is assumed to be strongly convergent; (2) the step size is supposed to always equal one after finite iterations; (3) the sufficient condition for the superlinear convergence is rather strong.

In this paper, we present an improved sequential quadratic programming algorithm for the minimax problem (1.1). In this algorithm, a main search direction is obtained by solving a quadratic program which always has a solution. In order to avoid the Maratos effect, unlike ([23]), a correction direction is yielded by solving a system of linear equation. Under mild conditions without the strict complementarity, global and superlinear convergence can be obtained. Finally, some preliminary numerical experiments are reported.

The balance of this paper is organised as follows. The algorithm and its properties are presented in Section 2. Global and superlinear convergence are analysed in Section 3 
and Section 4, respectively. Numerical results are reported in Section 5. Section 6 is devoted to final remarks.

\section{Algorithm}

For convenience of presentation, for a given $x \in R^{n}$, we use the following notation throughout the remainder of this paper

$$
\begin{aligned}
f(x) & =\left(f_{j}(x), j \in I\right)^{T}, & I(x) & =\left\{j \in I: f_{j}(x)=F(x)\right\}, \\
g_{j}(x) & =\nabla f_{j}(x), j \in I, & & g(x)=\left(g_{j}(x), j \in I\right) .
\end{aligned}
$$

We assume that the following assumptions hold in this paper.

(H1) Functions $f_{j}(j \in I)$ are all at least first order continuously differentiable.

(H2) Vectors $\left\{\left(\begin{array}{c}g_{j}(x) \\ -1\end{array}\right), j \in I(x)\right\}$ in $R^{n+1}$ are linearly independent.

REMARK 1. It is easy to prove that (H2) is equivalent to the following condition: For each $t \in I(x)$, vectors $\left\{g_{j}(x)-g_{t}(x), j \in I(x) \backslash\{t\}\right\}$ are linearly independent. This can also be illustrated from the following example: Let $I\left(x^{0}\right)=\{1,2,3\}$ and

$$
g_{1}\left(x^{0}\right)=\left(\begin{array}{l}
1 \\
0
\end{array}\right), g_{2}\left(x^{0}\right)=\left(\begin{array}{l}
2 \\
2
\end{array}\right), g_{3}\left(x^{0}\right)=\left(\begin{array}{l}
0 \\
1
\end{array}\right)
$$

for some $x^{0} \in R^{n}$. Obviously,

$$
\left\{\left(\begin{array}{c}
g_{j}\left(x^{0}\right) \\
-1
\end{array}\right), j \in I\left(x^{0}\right)\right\}
$$

are linearly independent and $\left\{g_{j}\left(x^{0}\right)-g_{t}\left(x^{0}\right), j \in I\left(x^{0}\right) \backslash\{t\}\right\}$ are linearly independent for each $t \in I\left(x^{0}\right)$.

Let $x^{k} \in R^{n}$ be a given iteration point. Based on (H2), we use the following pivoting operation to generate an $\varepsilon$-active constraint subset $I_{k} \supseteq I\left(x^{k}\right)$ such that the matrix $A_{k} \triangleq\left(\left(\begin{array}{c}g_{j}\left(x^{k}\right) \\ -1\end{array}\right), j \in I_{k}\right)$ is of full column rank.

Pivoting OPERATION POP.

STEP (i) Select an initial parameter $\varepsilon=\varepsilon_{k-1}>0$.

$\operatorname{STEP}(i i) \quad$ Generate the $\varepsilon$-active constraint subset $I\left(x^{k}, \varepsilon\right)$ and matrix $M_{k}$ by

$$
I\left(x^{k}, \varepsilon\right)=\left\{j \in I: \quad-\varepsilon \leqslant f_{j}\left(x^{k}\right)-F\left(x^{k}\right) \leqslant 0\right\}, \quad M_{k}=\left(\left(\begin{array}{c}
g_{j}\left(x^{k}\right) \\
-1
\end{array}\right), j \in I\left(x^{k}, \varepsilon\right)\right) .
$$

$\operatorname{STEP}(\mathrm{iii})$ If $\operatorname{det}\left(M_{k}^{T} M_{k}\right) \geqslant \varepsilon$, set $I_{k}=I\left(x^{k}, \varepsilon\right), A_{k}:=M_{k}$ and $\varepsilon_{k}=\varepsilon$, stop; otherwise set $\varepsilon:=(1 / 2) \varepsilon$ and repeat Step (ii). 
In order to show some beneficial properties of the pivoting operation above, which is helpful for discussing the convergence of our algorithm, we present the following lemma, and its proof is similar to [3, Lemmata 1.1 and 2.8], so it is omitted here.

Lemma 2.1. Suppose that (H1) and (H2) hold, and let $x^{k} \in R^{n}$. Then

(i) the pivoting operation can be finished in a finite number of computations, that is, there is no infinite times of loop between Step (ii) and Step (iii);

(ii) if a sequence $\left\{x^{k}\right\}$ of points has an accumulation point, then there exists an $\bar{\varepsilon}>0$ such that the associated sequence $\left\{\varepsilon_{k}\right\}$ of parameters generated by pivoting operation satisfies $\varepsilon_{k} \geqslant \bar{\varepsilon}$ for all $k$.

For a given iteration point $x^{k} \in R^{n}$ and a symmetric positive matrix $H_{k}=H\left(x^{k}\right)$ (How to choose $H_{k}$ will be discussed much later), we introduce a new quadratic program as follows:

$$
\begin{array}{ll}
\text { minimize } & z+\frac{1}{2} d^{T} H_{k} d \\
\text { such that } f_{j}\left(x^{k}\right)+g_{j}\left(x^{k}\right)^{T} d-F\left(x^{k}\right) \leqslant z, \quad j \in I_{k} .
\end{array}
$$

To describe the main characters of (2.3), we give two lemmas as follows.

LEMMA 2.2. Suppose that the matrix $H_{k}$ is symmetric positive definite. Then

(i) (2.3) has a unique optimal solution;

(ii) $\left(z_{k}, d^{k}\right)$ is an optimal solution of (2.3) if and only if it is a Karush-KuhnTucker point of (2.3).

It is not difficult to finish this proof, and is omitted.

LEMMA 2.3. Suppose that the assumptions (H1) and (H2) hold, and $\left(z_{k}, d^{k}\right)$ is an optimal solution of (2.3). Then

(i) $z_{k}+(1 / 2)\left(d^{k}\right)^{T} H_{k} d^{k} \leqslant 0, \quad z_{k} \leqslant 0 ; \quad d^{k}=0 \Leftrightarrow z_{k}=0$;

(ii) $d^{k}=0 \Leftrightarrow x^{k}$ is a stationary point of $(\mathrm{P})$;

(iii) if $d^{k} \neq 0$, then $z_{k}<0$, moreover, $d^{k}$ is a descent direction of $F(x)$ at point $x^{k}$.

Proof: (i) From the fact that $(0,0)$ is a feasible solution of $(2.3)$ and $H_{k}$ is positive definite, one has

$$
z_{k}+\frac{1}{2}\left(d^{k}\right)^{T} H_{k} d^{k} \leqslant 0, \quad z_{k} \leqslant-\frac{1}{2}\left(d^{k}\right)^{T} H_{k} d^{k} \leqslant 0 .
$$

If $d^{k}=0$, then from the constraints of (2.3) we have

$$
F\left(x^{k}\right)-f_{j}\left(x^{k}\right)+z_{k} \geqslant 0, \quad j \in I_{k} .
$$

In view of $\phi \neq I\left(x^{k}\right) \subseteq I_{k}$, one has $z_{k} \geqslant 0$. Since that $z_{k} \leqslant 0$, we have $z_{k}=0$. Conversely, if $z_{k}=0$, then $(1 / 2)\left(d^{k}\right)^{T} H_{k} d^{k}=(1 / 2)\left(d^{k}\right)^{T} H_{k} d^{k}+z_{k} \leqslant 0$, taking into account the positive definite property of $H_{k}$, one has $d^{k}=0$. 
(ii) In view of Lemma 2.2 (ii), we know that the optimal solution $\left(z_{k}, d^{k}\right)$ of (2.3) is a Karush-Kuhn-Tucker point of (2.3), then there exists a corresponding Karush-KuhnTucker multiplier vector $\lambda^{k}=\left(\lambda_{j}^{k}, j \in I_{k}, 0_{I \backslash I_{k}}\right)$ such that

$$
\left\{\begin{array}{l}
\left(\begin{array}{c}
1 \\
H_{k} d^{k}
\end{array}\right)+\sum_{j=1}^{m} \lambda_{j}^{k}\left(\begin{array}{c}
-1 \\
g_{j}\left(x^{k}\right)
\end{array}\right)=0, \\
f_{j}\left(x^{k}\right)+g_{j}\left(x^{k}\right)^{T} d^{k}-F\left(x^{k}\right)-z_{k} \leqslant 0, \quad j \in I_{k}, \\
\left(f_{j}\left(x^{k}\right)+g_{j}\left(x^{k}\right)^{T} d^{k}-F\left(x^{k}\right)-z_{k}\right) \lambda_{j}^{k}=0, \quad j \in I_{k}, \\
\lambda_{j}^{k} \geqslant 0, j \in I_{k} ; \quad \lambda_{j}^{k}=0, \quad j \in I \backslash I_{k} .
\end{array}\right.
$$

If $d^{k}=0$, then we get $z_{k}=0$ from Lemma 2.3 (i), and we further have from (2.4)

$$
\left\{\begin{array}{l}
\sum_{j=1}^{m} \lambda_{j}^{k} g_{j}\left(x^{k}\right)=0, \quad \sum_{j=1}^{m} \lambda_{j}^{k}=1, \\
f_{j}\left(x^{k}\right)-F\left(x^{k}\right) \leqslant 0, \quad j \in I, \\
\left(f_{j}\left(x^{k}\right)-F\left(x^{k}\right)\right) \lambda_{j}^{k}=0, \quad \lambda_{j}^{k} \geqslant 0, \quad j \in I .
\end{array}\right.
$$

Hence $x^{k}$ is a stationary point of $(\mathrm{P})$ from (1.3).

Conversely, if $x^{k}$ is a stationary point of (P), then $z_{k}:=0$ together $d^{k}:=0$ satisfies (2.4) and $(0,0)$ is the unique optimal solution of (2.3) from Lemma 2.2. Therefore $d^{k}=0$.

(iii) Using $z_{k}+(1 / 2)\left(d^{k}\right)^{T} H_{k} d^{k} \leqslant 0, d^{k} \neq 0$ and the positive definite property of the matrix $H_{k}$, we know that $z_{k}<0$ holds. Furthermore, in view of the constraints of (2.3), one gets

$$
g_{j}\left(x^{k}\right)^{T} d^{k} \leqslant z_{k}+F\left(x^{k}\right)-f_{j}\left(x^{k}\right)=z_{k}<0, \quad j \in I\left(x^{k}\right) .
$$

On the other hand, it is easy to know that the directional derivative $F^{\prime}(x ; d)$ of $F(x)$ at point $x$ along direction $d$ can be expressed as

$$
F^{\prime}(x ; d)=\lim _{\lambda \rightarrow 0^{+}} \frac{F(x+\lambda d)-F(x)}{\lambda}=\max \left\{g_{j}(x)^{T} d, \quad j \in I(x)\right\} .
$$

Thus

$$
F^{\prime}\left(x^{k} ; d^{k}\right) \leqslant z_{k}<0
$$

and $d^{k}$ is a descent direction of $F(x)$ at point $x^{k}$. The whole proof is completed.

Now we give the details of our algorithm as follows.

Algorithm A.

Parameters: $\varepsilon_{-1}>0, \quad \tau \in(2,3), \quad \alpha \in(0,0.5), \quad \beta \in(0,1), \quad \gamma<0$.

Data: $x^{0} \in R^{n}$, a symmetric positive definite matrix $H_{0} \in R^{n \times n}$ (Usually, $H_{0}$ is chosen as a unity matrix). 
STEP 0. Initialisation: Let $k:=0$.

STEP 1. Pivoting operation: Set parameter $\varepsilon=\varepsilon_{k-1}$, generate an active constraint set $I_{k}$ by the pivoting operation and let $\varepsilon_{k}$ be the corresponding termination parameter.

STEP 2. Quadratic programming: Solve (2.3) to get a solution $\left(z_{k}, d^{k}\right)$ with the corresponding Karush-Kuhn-Tucker multiplier vector $\lambda_{I_{k}}^{k}=\left(\lambda_{j}^{k}, j \in I_{k}\right)$. If $d^{k}=0$, then $x^{k}$ is a stationary point of problem (P) and stop; otherwise, enter Step 3.

STEP 3. Generate a correction direction $\tilde{d}^{k}$ : Compute direction $\widetilde{d}^{k}$ by solving the following system of linear equations

$$
\left[\begin{array}{cc}
\widetilde{H}_{k} & A_{k} \\
A_{k}^{T} & 0
\end{array}\right]\left[\begin{array}{c}
\tilde{t} \\
\tilde{\gamma}
\end{array}\right]=\left[\begin{array}{c}
0 \\
-\left\|d^{k}\right\|^{\tau} e-\tilde{f}_{k}
\end{array}\right],
$$

where

$$
\widetilde{H}_{k}=\left[\begin{array}{cc}
H_{k} & 0 \\
0 & 1
\end{array}\right], \tilde{t}=\left[\begin{array}{l}
\tilde{d} \\
\tilde{z}
\end{array}\right], e=(1,1, \ldots, 1)^{T} \in R^{\left|I_{k}\right|}
$$

and $\widetilde{f}_{k}=\left(\tilde{f}_{j}^{k}, j \in I_{k}\right), \widetilde{f}_{j}^{k}=f_{j}\left(x^{k}+d^{k}\right)-f_{j}\left(x^{k}\right)-g_{j}\left(x^{k}\right)^{T} d^{k}, \quad j \in I_{k}$. If $\left\|\widetilde{d}^{k}\right\| \geqslant\left\|d^{k}\right\|$, set $\widetilde{d}^{k}=0$.

STEP 4. Perform line search: Compute the step size $t_{k}$, the first number $t$ of the sequence $\left\{1, \beta, \beta^{2}, \ldots\right\}$ satisfying

$$
F\left(x^{k}+t d^{k}+t^{2} \tilde{d}^{k}\right) \leqslant \max _{l=0,1,2} F\left(x^{k-1}\right)-\alpha t\left(d^{k}\right)^{T} H_{k} d^{k} .
$$

STEP 5 Update: Generate a new symmetric positive definite matrix $H_{k+1}$ using the formula proposed by [2], set $x^{k+1}=x^{k}+t_{k} d^{k}+t_{k}^{2} \widetilde{d}^{k}$ and $k:=k+1$. Go back to Step 1 .

To show that the algorithm is well defined, we introduce the following lemma.

Lemma 2.4. The line search in Step 4 can be carried out if $d^{k} \neq 0$, that is, there exists $\bar{t}_{k}>0$ such that (2.9) bolds.

Proof: By contradiction, we assume that (2.9) does not hold for $\lambda=\beta^{j}$, $j=1,2, \ldots$, then from (2.7), (2.6), $\alpha \in(0,0.5), \beta \in(0,1)$ and Lemma 2.3 (i), we have

$$
\begin{aligned}
z_{k} & \geqslant F^{\prime}\left(x^{k} ; d^{k}\right)=\lim _{j \rightarrow \infty} \frac{F\left(x^{k}+\beta^{j} d^{k}\right)-F\left(x^{k}\right)}{\beta^{j}}=\lim _{j \rightarrow \infty} \frac{F\left(x^{k}+\beta^{j} d^{k}+\beta^{2 j} \tilde{d}^{k}\right)-F\left(x^{k}\right)}{\beta^{j}} \\
& \geqslant \lim _{j \rightarrow \infty} \frac{F\left(x^{k}+\beta^{j} d^{k}+\beta^{2 j} \widetilde{d}^{k}\right)-\max _{l=0,1,2} F\left(x^{k-l}\right)}{\beta^{j}} \\
& \geqslant-\lim _{j \rightarrow \infty} \alpha\left(d^{k}\right)^{T} H_{k} d^{k}>-\frac{1}{2}\left(d^{k}\right)^{T} H_{k} d^{k} \geqslant z_{k},
\end{aligned}
$$

which is a contradiction. The proof is completed. 


\section{Global Convergence}

In this section, we shall establish the global convergence of the proposed algorithm. If the solution $d^{k}$ generated at Step 2 equals zero, then Algorithm $\mathrm{A}$ stops at $x^{k}$, and from Lemma 2.3 (ii) we know that $x^{k}$ is a stationary point of the problem (P). And if $d^{k} \neq 0$, one knows from Lemma 2.3 (iii) that $d^{k}$ is a descent direction of $F(x)$ at point $x^{k}$.

We further assume that an infinite sequence $\left\{x^{k}\right\}$ of points is generated by Algorithm $\mathrm{A}$, and the consequent task is to show that every accumulation point $x^{*}$ of $\left\{x^{k}\right\}$ is a stationary point of problem $(\mathrm{P})$. Firstly, the following assumption is necessary in the rest of this paper.

(H3) The sequence $\left\{H_{k}\right\}$ of matrices is uniformly positive definite, that is, there exist two positive constants $a$ and $b$ such that

$$
a\|d\|^{2} \leqslant d^{T} H_{k} d \leqslant b\|d\|^{2}, \quad \forall d \in R^{n}, \forall k .
$$

(H4) For any $x^{0} \in R^{n}$, the set $\Omega=\left\{x \in R^{n}: f(x) \leqslant f\left(x^{0}\right)\right\}$ is compact.

In the rest of this paper, we suppose that $x^{*}$ is a given accumulation point of $\left\{x^{k}\right\}$, therefore, in view of $I_{k}$ being the subset of the fixed and finite set $I$ and Lemma 2.1, we may assume without loss of generality that there exist an infinite index set $K$ and a constant $\bar{\varepsilon}>0$ such that

$$
x^{k} \rightarrow x^{*}, I\left(x^{k}\right) \equiv I_{*} \neq \phi, \quad I_{k} \equiv I^{\prime}, \forall k \in K ; \quad \varepsilon_{k} \geqslant \bar{\varepsilon}, \forall k .
$$

LEMMA 3.1. ([23]) The sequence $\left\{x^{k}\right\}$ is bounded and the sequence $\left\{t_{k} d^{k}\right\}$ and $\left\{x^{k+1}-x^{k}\right\}$ both converge to zero.

LEMMA 3.2. Suppose that the assumptions (H1)-(H3) hold. Then

(i) the sequences $\left\{z_{k}, k \in K\right\},\left\{d^{k}, k \in K\right\}$ and $\left\{\widetilde{d}^{k}, k \in K\right\}$ are all bounded;

(ii) $\lim _{k \in K} d^{k}=\lim _{k \in K} \widetilde{d}^{k}=0, \lim _{k \in K} z_{k}=0$.

Proof: (i) Due to the fact that $(0,0)$ is a feasible solution of $(2.3)$, combining assumption (H3) and the constraints of (2.3), we have

$$
\begin{aligned}
0 & \geqslant z_{k}+\frac{1}{2}\left(d^{k}\right)^{T} H_{k} d^{k} \geqslant f_{j}\left(x^{k}\right)+g_{j}\left(x^{k}\right)^{T} d^{k}-F\left(x^{k}\right)+\frac{1}{2}\left(d^{k}\right)^{T} H_{k} d^{k} \\
& =g_{j}\left(x^{k}\right)^{T} d^{k}+\frac{1}{2}\left(d^{k}\right)^{T} H_{k} d^{k} \geqslant-\left\|g_{j}\left(x^{k}\right)\right\| \cdot\left\|d^{k}\right\|+\frac{1}{2} a\left\|d^{k}\right\|^{2}, \quad \forall j \in I\left(x^{k}\right), \forall k .
\end{aligned}
$$

These inequalities show that $\left\{z_{k}, k \in K\right\}$ and $\left\{d^{k}, k \in K\right\}$ are all bounded. Taking into account the definition of $\widetilde{d^{k}}$ at Step 3 of Algorithm A, we can conclude that $\left\{\widetilde{d}^{k}, k \in K\right\}$ is bounded.

(ii) Similar to the proof of $\left[\mathbf{2 3}\right.$, Theorem 3.1], we can prove $\lim _{k \in K} d^{k}=0$. This shows that conclusion (ii) is true. 
LEMMA 3.3. The whole multiplier sequence $\left\{\lambda^{k}=\left(\lambda_{I_{k}}^{k}, 0_{I \backslash I_{k}}\right)\right\}$ is bounded.

Proof: From (2.4) we get $\sum_{j=1}^{m} \lambda_{j}^{k}=1$ and $\lambda_{j}^{k} \geqslant 0, j \in I$. Thus sequence $\left\{\lambda^{k}\right\}$ is bounded.

Now, it is sufficient to establish the following globally convergent theorem for the proposed algorithm.

THEOREM 3.1. Suppose that the assumptions (H1)-(H4) hold, then the proposed Algorithm $A$ either stops at a stationary point of problem $(\mathrm{P})$ in a finite number of iterations, or generates an infinite sequence $\left\{x^{k}\right\}$ of points such that each accumulation $x^{*}$ of $\left\{x^{k}\right\}$ is a stationary point of $(\mathrm{P})$.

The proof is similar to the one of [23, Theorem 3.1].

\section{RATE OF CONVERGENCE}

Firstly, we give a proposition as follows, which would be useful in the rest discussions.

PROpOSITION 4.1. Suppose that the assumptions (H1) and (H2) hold. Then the multiplier vector corresponding to a stationary point $\tilde{x}$ of $(\mathrm{P})$ is unique.

Proof: Suppose that $\tilde{\lambda}, \tilde{\mu}$ are two multiplier vectors corresponding to a same stationary point $\widetilde{x}$. Then we have from (1.3)

$$
\sum_{j \in I(\tilde{x})} \tilde{\lambda}_{j}\left(\begin{array}{c}
g_{j}(\tilde{x}) \\
-1
\end{array}\right)=\left(\begin{array}{c}
0 \\
-1
\end{array}\right), \sum_{j \in I(\tilde{x})} \tilde{\mu}_{j}\left(\begin{array}{c}
g_{j}(\tilde{x}) \\
-1
\end{array}\right)=\left(\begin{array}{c}
0 \\
-1
\end{array}\right), \quad \tilde{\lambda}_{I \backslash I(\tilde{x})}=\tilde{\mu}_{I \backslash I(\tilde{x})}=0
$$

These two equations give $\sum_{j \in I(\tilde{x})}\left(\tilde{\mu}_{j}-\tilde{\lambda}_{j}\right)\left(\begin{array}{c}g_{j}(\tilde{x}) \\ -1\end{array}\right)=0$. Then $\tilde{\lambda}=\tilde{\mu}$ follows from (H2).

THEOREM 4.1. Suppose that the assumptions (H1)-(H4) hold. Then

$$
\lim _{k \rightarrow \infty} d^{k}=\lim _{k \rightarrow \infty} \hat{d}^{k}=0, \lim _{k \rightarrow \infty} z_{k}=0 .
$$

The proof is similar to the one of [23, Theorem 3.1].

In order to obtain the superlinearly convergent rate of the proposed algorithm, from Proposition 4.1 above, we further suppose that the following assumption holds.

(i) The functions $f_{j}(x)(j \in I)$ are all twice continuously differentiable for any $x \in R^{n}$

(ii) The sequence $\left\{x^{k}\right\}$ generated by Algorithm A possesses an accumulation point $x^{*}$ with the corresponding unique multipliers $\mu^{*}$ (by Theorem $3.1, x^{*}$ is a stationary point of problem (P)), such that the stationary point pair 
$\left(x^{*}, \mu^{*}\right)$ of problem (P) satisfies the following strong second-order sufficiency conditions for some index $t_{0} \in I_{*}^{+}$

$d^{T} \nabla_{x x}^{2} L\left(x^{*}, \mu^{*}\right) d>0, \quad \forall d \in R^{n}: d \neq 0,\left(g_{j}\left(x^{*}\right)-g_{t_{0}}\left(x^{*}\right)\right)^{T} d=0, j \in I_{*}^{+}$,

where

$$
\nabla_{x x}^{2} L\left(x^{*}, \mu^{*}\right)=\sum_{j \in I} \mu_{j}^{*} \nabla^{2} f_{j}\left(x^{*}\right)=\sum_{j \in I_{+}^{+}} \mu_{j}^{*} \nabla^{2} f_{j}\left(x^{*}\right), I_{*}^{+} \triangleq\left\{j \in I: \mu_{j}^{*}>0\right\} .
$$

REMARK 2. Since the assumption (H5) above does not include the so-called strict complementary condition, namely $\mu_{j}^{*}>0$ for all $j \in I\left(x^{*}\right)$, which is assumed to be hold in assumption in [23, A5] and [22, Assumption 3.4], it is a weaker hypothesis.

Now we prove that $x^{*}$ is an isolated stationary point of $(\mathrm{P})$ under certain conditions.

Lemma 4.1. Suppose that assumptions (H2) and (H5) hold. Then $x^{*}$ is an isolated stationary point of $(\mathrm{P})$.

The proof is similar to the one of [6, Theorem 1.2.5].

THEOREM 4.2. Suppose that the assumptions (H2)-(H5) hold. Then $\lim _{k \rightarrow \infty} x^{k}=x^{*}$.

Proof: From Lemma 4.1, we know that $x^{*}$ is an isolated stationary point of (P). Furthermore, one can conclude $x^{*}$ is an isolated limit point of $\left\{x^{k}\right\}$ and this together with Theorem 4.1 (ii) implies $\lim _{k \rightarrow \infty} x^{k}=x^{*}$ (see [6, Theorem 1.1.5]).

LEMMA 4.2. Under all the above-mentioned assumptions, when $k$ is sufficiently large, the matrix

$$
M_{k} \stackrel{\text { def }}{=}\left[\begin{array}{cc}
\widetilde{H}_{k} & A_{k} \\
A_{k}^{T} & 0
\end{array}\right] .
$$

is nonsingular, furthermore, there exists a constant $C>0$ such that $\left\|M_{k}^{-1}\right\| \leqslant C$.

The proof of Lemma 4.2 is similar to that of [6, Lemma 2.2.2], and is omitted.

Lemma 4.3. Suppose that the assumptions (H2) and (H3) hold. Then $\left\|\widetilde{d}^{k}\right\|$ $=O\left(\left\|d^{k}\right\|^{2}\right)$.

Proof: Taking into account Taylor expansion, the definition of $\tilde{f}_{j}^{k}$ and Theorem 4.1 (i), we obtain

$$
\hat{f}_{j}^{k}=f_{j}\left(x^{k}+d^{k}\right)-f_{j}\left(x^{k}\right)-g_{j}\left(x^{k}\right)^{T} d^{k}=O\left(\left\|d^{k}\right\|^{2}\right) .
$$

So, by using the Lemma $4.2, \tau \in(2,3)$ and (2.8), we have $\left\|\widetilde{d^{k}}\right\|=O\left(\left\|d^{k}\right\|^{2}\right)$.

Lemma 4.4. If assumptions (H2)-(H5) are all satisfied, then the Karush-KuhnTucker multiplier $\lambda_{I_{k}}^{k}$ of (2.3) corresponding to $\left(z_{k}, d^{k}\right)$ satisfies $\lim _{k \rightarrow \infty} \lambda^{k}=\mu^{*}$ with $\lambda^{k}=\left(\lambda_{I_{k}}^{k}, 0_{I \backslash I_{k}}\right)$. 
Proof: We assume by contradiction that $\lim _{k \rightarrow \infty} \lambda^{k} \neq \mu^{*}$, then there exists an infinite subset $K$ and a constant $\bar{a}>0$ such that

$$
\left\|\lambda^{k}-\mu^{*}\right\| \geqslant \bar{a}, k \in K \text {. }
$$

In view of $\lim _{k \rightarrow \infty} x^{k}=x^{*}$ and the boundedness of $\left\{\lambda^{k}\right\}$, there exists another infinite set $K^{\prime} \subseteq K$ such that

$$
x^{k} \rightarrow x^{*},\left\|\lambda^{k}-\mu^{*}\right\| \geqslant \bar{a}, \quad \lambda^{k} \rightarrow \lambda^{*}, k \in K^{\prime} \subseteq K .
$$

Taking into account of Theorem 4.1 and passing to the limit $k \in K^{\prime}$ and $k \rightarrow \infty$ in (2.4), we have

$$
\left\{\begin{array}{l}
\left(\begin{array}{l}
1 \\
0
\end{array}\right)+\sum_{j=1}^{m} \lambda_{j}^{*}\left(\begin{array}{c}
-1 \\
g_{j}\left(x^{*}\right)
\end{array}\right)=0, \\
f_{j}\left(x^{*}\right)-F\left(x^{*}\right) \leqslant 0, \quad\left(f_{j}\left(x^{*}\right)-F\left(x^{*}\right)\right) \lambda_{j}^{*}=0, \quad \lambda_{j}^{*} \geqslant 0, \quad j \in I .
\end{array}\right.
$$

From above we know that $\left(x^{*}, \lambda^{*}\right)$ is a stationary point pair of $(\mathrm{P})$, thus $\lambda^{*}=\mu^{*}$ (since the multiplier vector is unique), which contradicts (4.1). So the whole proof is finished.

In order to obtain the superlinearly convergent rate of the proposed algorithm, first of all, we should guarantee that the step size unit is accepted by the line search for $k$ large enough. For this purpose, the following additional assumptions are necessary.

(H6) Suppose that $\left\|\left(\nabla_{x x}^{2} L\left(x^{k}, \lambda^{k}\right)-H_{k}\right) d^{k}\right\|=o\left(\left\|d^{k}\right\|\right)$.

Theorem 4.3. Under all above assumptions (H2)-(H6), the step size in Algorithm $A$ always equals to one, that is, $t_{k} \equiv 1$, if $k$ is sufficient large.

Proof: It is sufficient to show that (2.9) holds for $t=1$. Firstly, in view of Taylor expansion, Theorems 4.1, 4.2 and Lemma 4.2, we get

$$
\begin{aligned}
f_{i}\left(x^{k}+d^{k}+\tilde{d}^{k}\right) & =f_{i}\left(x^{k}+d^{k}\right)+g_{i}\left(x^{k}+d^{k}\right)^{T} \tilde{d^{k}}+O\left(\left\|\tilde{d^{k}}\right\|^{2}\right) \\
& =f_{i}\left(x^{k}+d^{k}\right)+g_{i}\left(x^{k}\right)^{T} \tilde{d}^{k}+O\left(\left\|d^{k}\right\|^{3}\right), i \in J_{k} .
\end{aligned}
$$

Where $J_{k}=\left\{i \mid f_{j}\left(x^{k}\right)+g_{j}\left(x^{k}\right)^{T} d^{k}-F\left(x^{k}\right)=z_{k}\right\}$.

From (2.8), we also have

$$
A_{k}^{T}\left(\begin{array}{c}
\tilde{d}^{k} \\
\tilde{z}_{k}
\end{array}\right)=-\left\|d^{k}\right\|^{\top} e-\tilde{f}^{k}, \quad g_{i}\left(x^{k}\right)^{T} \tilde{d}^{k}=\tilde{z}_{k}-\left\|d^{k}\right\|^{\top}-\tilde{f}_{i}^{k}, i \in J_{k} \subseteq I_{k} .
$$

Hence from (4.2), (4.3) and the definition of $\tilde{f}_{i}^{k}$, we have

$$
\begin{aligned}
f_{i}\left(x^{k}+d^{k}+\tilde{d}^{k}\right) & =f_{i}\left(x^{k}+d^{k}\right)+\tilde{z}_{k}-\left\|d^{k}\right\|^{\tau}-\tilde{f}_{i}^{k}+O\left(\left\|d^{k}\right\|^{3}\right) \\
& =\tilde{z}_{k}-\left\|d^{k}\right\|^{\tau}+f_{i}\left(x^{k}\right)+g_{i}\left(x^{k}\right)^{T} d^{k}+O\left(\left\|d^{k}\right\|^{3}\right) \\
& =F\left(x^{k}\right)+z_{k}+\tilde{z}_{k}-\left\|d^{k}\right\|^{\tau}+O\left(\left\|d^{k}\right\|^{3}\right), \quad i \in J_{k} \subseteq I_{k}
\end{aligned}
$$


So

$$
f_{j}\left(x^{k}+d^{k}+\widetilde{d}^{k}\right)=F\left(x^{k}\right)+z_{k}+\widetilde{z}_{k}-\left\|d^{k}\right\|^{\tau}+O\left(\left\|d^{k}\right\|^{3}\right), j \in J_{k} .
$$

Thus from the two formulas above, we obtain

$$
f_{i}\left(x^{k}+d^{k}+\tilde{d}^{k}\right)=f_{j}\left(x^{k}+d^{k}+\tilde{d}^{k}\right)+O\left(\left\|d^{k}\right\|^{3}\right), \forall i, j \in J_{k} .
$$

Now we shall show that for $k$ large enough

$$
F\left(x^{k}+d^{k}+\tilde{d}^{k}\right)=f_{j}\left(x^{k}+d^{k}+\tilde{d}^{k}\right)+O\left(\left\|d^{k}\right\|^{3}\right), \forall j \in J_{k} .
$$

In view of $I\left(x^{k}+d^{k}+\widetilde{d}^{k}\right) \subseteq I\left(x^{*}\right), J_{k} \subseteq I\left(x^{*}\right) \subseteq I_{k}$ for $k$ large enough, one has

$$
F\left(x^{k}+d^{k}+\widetilde{d}^{k}\right)=\max \left\{f_{i}\left(x^{k}+d^{k}+\widetilde{d}^{k}\right), i \in J_{k}, f_{i}\left(x^{k}+d^{k}+\widetilde{d}^{k}\right), i \in I\left(x^{*}\right) \backslash J_{k}\right\} .
$$

CASE A. If there exists an index $j_{0} \in J_{k}$ such that $F\left(x^{k}+d^{k}+\widetilde{d}^{k}\right)=f_{j_{0}}\left(x^{k}+d^{k}+\widetilde{d}^{k}\right)$, then from (4.4) we have

$$
F\left(x^{k}+d^{k}+\widetilde{d}^{k}\right)=f_{j}\left(x^{k}+d^{k}+\tilde{d}^{k}\right)+O\left(\left\|d^{k}\right\|^{3}\right), \forall j \in J_{k} .
$$

CASE B. If $f_{j}\left(x^{k}+d^{k}+\widetilde{d}^{k}\right)<F\left(x^{k}+d^{k}+\widetilde{d}^{k}\right), \forall j \in J_{k}$, then there exists an index $j_{1} \in I\left(x^{*}\right) \backslash J_{k} \subseteq I_{k} \backslash J_{k}$ such that

$$
F\left(x^{k}+d^{k}+\tilde{d}^{k}\right)=f_{j_{1}}\left(x^{k}+d^{k}+\tilde{d}^{k}\right) .
$$

From Taylor expansion, we have

$$
f_{j_{1}}\left(x^{k}+d^{k}+\tilde{d}^{k}\right)=f_{j_{1}}\left(x^{k}+d^{k}\right)+g_{j_{1}}\left(x^{k}\right)^{T} \tilde{d}^{k}+O\left(\left\|d^{k}\right\|^{3}\right),
$$

In view of (2.8), similar to (4.3), one gets

$$
g_{j_{1}}\left(x^{k}\right)^{T} \tilde{d}^{k}=\tilde{z}_{k}-\left\|d^{k}\right\|^{\tau}-\tilde{f}_{j_{1}}^{k}, j_{1} \in I\left(x^{*}\right) \backslash J_{k} \subseteq I_{k} \backslash J_{k} .
$$

Thus

$$
\begin{aligned}
f_{j_{1}}\left(x^{k}+d^{k}+\widetilde{d}^{k}\right)= & f_{j_{1}}\left(x^{k}+d^{k}\right)+\widetilde{z}_{k}-\left\|d^{k}\right\|^{\top}+f_{j_{1}}\left(x^{k}\right)+g_{j_{1}}\left(x^{k}\right)^{T} d^{k} \\
& -f_{j_{1}}\left(x^{k}+d^{k}\right)+O\left(\left\|d^{k}\right\|^{3}\right) \\
= & f_{j_{1}}\left(x^{k}\right)+g_{j_{1}}\left(x^{k}\right)^{T} d^{k}+\widetilde{z_{k}}-\left\|d^{k}\right\|^{\top}+O\left(\left\|d^{k}\right\|^{3}\right) \\
< & F\left(x^{k}\right)+z_{k}+\widetilde{z}_{k}-\left\|d^{k}\right\|^{\tau}+O\left(\left\|d^{k}\right\|^{3}\right),
\end{aligned}
$$

that is

$$
F\left(x^{k}+d^{k}+\tilde{d}^{k}\right)<F\left(x^{k}\right)+z_{k}+\tilde{z}_{k}-\left\|d^{k}\right\|^{\tau}+O\left(\left\|d^{k}\right\|^{3}\right), i \in J_{k},
$$

combining

$$
f_{i}\left(x^{k}+d^{k}+\tilde{d}^{k}\right)=F\left(x^{k}\right)+z_{k}+\tilde{z}_{k}-\left\|d^{k}\right\|^{\tau}+O\left(\left\|d^{k}\right\|^{3}\right)
$$


imply that

$$
F\left(x^{k}+d^{k}+\tilde{d}^{k}\right)=f_{j}\left(x^{k}+d^{k}+\tilde{d}^{k}\right)+O\left(\left\|d^{k}\right\|^{3}\right), \forall j \in J_{k} .
$$

On the other hand, from Theorem 4.1 it is easy to get $J_{k} \subseteq I\left(x^{*}\right)$ for $k$ large enough. Thus, from (2.4), Lemma 3.2, Taylor expansion and Lemma 4.2, we get

$$
\begin{aligned}
& \sum_{j \in J_{k}} \lambda_{j}^{k}=1, \quad \lambda_{j}^{k} F\left(x^{k}+d^{k}+\tilde{d}^{k}\right)=\lambda_{j}^{k} f_{j}\left(x^{k}+d^{k}+\tilde{d}^{k}\right)+O\left(\left\|d^{k}\right\|^{3}\right), \quad j \in J_{k}, \\
& \begin{aligned}
F\left(x^{k}\right. & \left.+d^{k}+\tilde{d}^{k}\right) \\
& =\sum_{j \in J_{k}} \lambda_{j}^{k} F\left(x^{k}+d^{k}+\tilde{d}^{k}\right)=\sum_{j \in J_{k}} \lambda_{j}^{k} f_{j}\left(x^{k}+d^{k}+\tilde{d}^{k}\right)+O\left(\left\|d^{k}\right\|^{3}\right) \\
& =\sum_{j \in J_{k}} \lambda_{j}^{k}\left(f_{j}\left(x^{k}\right)+g_{j}\left(x^{k}\right)^{T}\left(d^{k}+\tilde{d}^{k}\right)+\frac{1}{2}\left(d^{k}\right)^{T} \nabla^{2} f_{j}\left(x^{k}\right) d^{k}\right)+o\left(\left\|d^{k}\right\|^{2}\right) .
\end{aligned}
\end{aligned}
$$

Also, from (2.4) and Lemma 4.2, one has

$$
\sum_{j \in J_{k}} \lambda_{j}^{k} g_{j}\left(x^{k}\right)^{T}\left(d^{k}+\tilde{d}^{k}\right)=-\left(d^{k}\right)^{T} H_{k} d^{k}+o\left(\left\|d^{k}\right\|^{2}\right)
$$

and

$$
\sum_{j \in J_{k}} \lambda_{j}^{k} f_{j}\left(x^{k}\right) \leqslant \sum_{j \in J_{k}} \lambda_{j}^{k} F\left(x^{k}\right)=F\left(x^{k}\right) .
$$

So, from (4.5)-(4.7), (H3) and (H5), we have

$$
\begin{aligned}
& F\left(x^{k}+d^{k}+\tilde{d}^{k}\right) \\
& \quad \leqslant \max _{l=0,1,2} F\left(x^{k-l}\right)-\left(d^{k}\right)^{T} H_{k} d^{k}+\frac{1}{2}\left(d^{k}\right)^{T}\left(\sum_{j \in J_{k}} \lambda_{j}^{k} \nabla^{2} f_{j}\left(x^{k}\right)\right) d^{k}+o\left(\left\|d^{k}\right\|^{2}\right) \\
& \quad=\max _{l=0,1,2} F\left(x^{k-l}\right)-\frac{1}{2}\left(d^{k}\right)^{T} H_{k} d^{k}+\frac{1}{2}\left(d^{k}\right)^{T}\left(\sum_{j \in J_{k}} \lambda_{j}^{k} \nabla^{2} f_{j}\left(x^{k}\right)-H_{k}\right) d^{k}+o\left(\left\|d^{k}\right\|^{2}\right) \\
& \quad=\max _{l=0,1,2} F\left(x^{k-l}\right)-\alpha\left(d^{k}\right)^{T} H_{k} d^{k}+\left(\alpha-\frac{1}{2}\right)\left(d^{k}\right)^{T} H_{k} d^{k}+o\left(\left\|d^{k}\right\|^{2}\right) \\
& \quad \leqslant \max _{l=0,1,2} F\left(x^{k-l}\right)-\alpha\left(d^{k}\right)^{T} H_{k} d^{k}+\left(\alpha-\frac{1}{2}\right) a\left\|\left(d^{k}\right)\right\|^{2}+o\left(\left\|d^{k}\right\|^{2}\right) .
\end{aligned}
$$

Taking into account $\alpha \in(0,1 / 2)$, we have for $k$ large enough

$$
F\left(x^{k}+d^{k}+\tilde{d}^{k}\right) \leqslant \max _{l=0,1,2} F\left(x^{k-l}\right)-\alpha\left(d^{k}\right)^{T} H_{k} d^{k},
$$

that is (2.9) holds for $t=1$ and $k$ large enough. So the whole proof is finished.

To analyse the superlinear convergence, we further give a lemma as follows. 
LEMMA 4.5. Suppose that the assumptions (H2)-(H5) hold and let

$$
R_{k}=R\left(x^{k}\right)=\left(g_{j}\left(x^{k}\right)-g_{t_{0}}\left(x^{k}\right), j \in J_{k} \backslash\left\{t_{0}\right\}\right), \quad P_{k}=E_{n}-R_{k}\left(R_{k}^{T} R_{k}\right)^{-1} R_{k}^{T} .
$$

Then for $k$ large enough the following matrix

$$
G_{k}=\left(\begin{array}{cc}
P_{k} \nabla_{x x}^{2} L\left(x^{*}, \mu^{*}\right) & R_{k} \\
R_{k}^{T} & 0
\end{array}\right)
$$

is nonsingular and there exists a constant $c$ such that $\left\|G_{k}^{-1}\right\| \leqslant c$.

The proof of this Lemma is similar to the one of Lemma 4.2, and is omitted.

THEOREM 4.4. Let the assumptions (H2)-(H6) be satisfied. Then the proposed algorithm $A$ is superlinearly convergent, that is, the sequence $\left\{x^{k}\right\}$ generated by algorithm A satisfies

$$
\left\|x^{k+1}-x^{*}\right\|=o\left(\left\|x^{k}-x^{*}\right\|\right) .
$$

The proof is similar to the one of [6, Theorem 2.2.3].

\section{NUMERICAL EXPERIMENTS}

In this section, we test some practical problems based on the proposed algorithm. The numerical experiments are implemented on MATLAB 6.5, under Windows XP and 1000MHZ CPU. The (2.3) and (2.8) are solved by the Optimisation Toolbox.

A slight modification of the Broyden-Fletcher-Goldfarb-Shanno formula, which is proposed in [2], is adopted in the algorithm.

$$
H_{k+1}=H_{k}-\frac{H_{k} s^{k}\left(s^{k}\right)^{T} H_{k}}{\left(s^{k}\right)^{T} H_{k} s^{k}}+\frac{\widetilde{y}^{k}\left(\widetilde{y}^{k}\right)^{T}}{\left(s^{k}\right)^{T} \widetilde{y}^{k}}, \quad(k \geqslant 0)
$$

where

$$
\begin{gathered}
s^{k}=x^{k+1}-x^{k}, \tilde{y}^{k}=y^{k}+a_{k}\left(\gamma_{k} s^{k}+\bar{A}_{k} \bar{A}_{k}^{T} s^{k}\right), \gamma_{k}=\min \left\{\left\|d_{0}^{k}\right\|^{2}, \xi\right\}, \xi \in(0,1) \\
y^{k}=\nabla_{x} L\left(x^{k+1}, \lambda^{k}\right)-\nabla_{x} L\left(x^{k}, \lambda^{k}\right), \bar{A}_{k}=\left(g_{j}\left(x^{k}\right), j \in I\left(x^{k}\right)\right), \\
\nabla_{x} L(x, \lambda)=\sum_{j \in I(x)} \lambda_{j} g_{j}(x), \\
a_{k}=\left\{\begin{array}{lll}
0, & \text { if } \quad\left(s^{k}\right)^{T} y^{k} \geqslant \delta\left\|s^{k}\right\|^{2}, \delta \in(0,1) ; \\
1, & \text { if } 0 \leqslant\left(s^{k}\right)^{T} y^{k}<\delta\left\|s^{k}\right\|^{2} ; \\
1+\frac{\gamma_{k}\left\|s^{k}\right\|^{2}-\left(s^{k}\right)^{T} y^{k}}{\gamma_{k}\left\|s^{k}\right\|^{2}+\left(s^{k}\right)^{T} \bar{A}_{k}\left(\bar{A}_{k}\right)^{T} s^{k}}, & \text { otherwise. }
\end{array}\right.
\end{gathered}
$$

During the numerical experiments, we set

$$
H_{0}=I, \quad \tau=2.5, \quad \beta=0.6, \quad \alpha=0.45, \quad \varepsilon_{-1}=2.1,
$$


where $I$ is an unity matrix. The test problem in Table 5.1 are selected from [21] and [19]. The initial points for the selected problems are as same as the ones in [21] and [19]. The columns of Table 5.1 have the following meanings: The prob column lists the test problem taken from [21] and [19]. The columns labelled $\mathrm{Ni}$ give the number of iterations required to solve the problem. The columns labelled objective, dnorm and eps denote the final objective value, the norm of $d^{k}$ and the step criterion threshold $\varepsilon$, respectively.

The detailed information of the solutions to the test problems is listed in the following Table 5.1. It can be seen from Table 5.1 that the proposed algorithm is effective, since it can successfully reach a near-optimal point for all the tested problems. However, we also observe that the numerical results reported here have more number of function evaluations than those reported in [21], in that our algorithm needs the PIVOTING technique which results in a reduction of the size of the quadratic programming subproblem.

Table 5.1 Numerical results

\begin{tabular}{|c|c|c|c|c|}
\hline \hline Prob & Ni & objective & dnorm & eps \\
\hline 1 & 10 & 1.9522 & $4.2478 \mathrm{e}-006$ & $0.1 \mathrm{e}-04$ \\
\hline 2 & 31 & 2.0000 & $1.9866 \mathrm{e}-015$ & $0.1 \mathrm{e}-04$ \\
\hline 3 & 11 & -44.0000 & $8.2939 \mathrm{e}-006$ & $0.1 \mathrm{e}-04$ \\
\hline 4 & 22 & 0.6164 & $8.7974 \mathrm{e}-006$ & $0.1 \mathrm{e}-04$ \\
\hline 5 & 15 & 3.5997 & $1.8327 \mathrm{e}-006$ & $0.1 \mathrm{e}-04$ \\
\hline 8 & 23 & 680.6301 & $1.5379 \mathrm{e}-006$ & $0.1 \mathrm{e}-04$ \\
\hline 9 & 34 & 24.3062 & $2.5866 \mathrm{e}-006$ & $0.1 \mathrm{e}-04$ \\
\hline Vardi-3 & 10 & -48.0158 & $4.5843 \mathrm{e}-008$ & $0.1 \mathrm{e}-04$ \\
\hline
\end{tabular}

\section{Concluding Remarks}

In this paper, we propose a nonmonotone descent sequential quadratic programming algorithm for nonlinear minimax problems. At each iteration, by solving a reduced quadratic programming subproblem, a main search direction is obtained. Then we correct the main search direction by solving a reduced system of linear equation. A nonmonotone line search is performed on $F(x)$ to obtain the next iteration point. Under weaker conditions without the strict complementarity, the global and one-step superlinear convergent properties are obtained. Preliminary numerical results also show that the proposed algorithm is effective.

\section{REFERENCES}

[1] A.R. Conn and Y. Li, 'An efficient algorithm for nonlinear minimax problems', Report CS-88-41, University of Waterloo, Waterloo, Ontario, Canada.

[2] J.F.A. De, O. Pantoja and D.Q. Mayne, 'Exact penalty function algorithm with simple updating of the penalty parameter', J. Optim. Theory Appl. 69 (1991), 441-467. 
[3] Z.-Y. Gao, G.-P. He and F. Wu, 'A method of sequential systems of linear equations with arbitary initial point', Sci. China Ser. $A 27$ (1997), 24-33.

[4] S.P. Han, 'A globally convergent method for nonlinear programming', J. Optim. Theory Appl. 22 (1977), 297-309.

[5] S.P. Han, 'Variable metric methods for minimizing a class of nondifferentiable functions', Math. Programming 20 (1981), 1-13.

[6] J.-B. Jian, Researches on superlinearly and quadratically convergent algorithms for nonlinearly constrained optimization, (Ph. D. Thesis) (School of Xi'an Jiaotong University, Xi'an, China, 2000).

[7] J.-B. Jian and C.-M. Tang, 'An SQP feasible descent algorithm for nonlinear inequality constrained optimization without strict complementarity', Comput. Math. Appl. 49 (2005), 223-238.

[8] J.-B. Jian, K.-C. Zhang and S.-J. Xue, 'A superlinearly and quadratically convergent SQP type feasible method for constrained optimization', Appl. Math. J. Chinese Univ. Ser. $B 15$ (2000), 319-331.

[9] L. Luskan, 'A compact variable metric algorithm for nonlinear minimax approximation', Computing 36 (1986), 355-373.

[10] D.Q. Mayne and E. Polak, 'A superlinearly convergent algorithm for constrained optimization problems', Math. Programming Stud. 16 (1982), 45-61.

[11] D.Q. Mayne, E. Polak and A. Sangiovanni-Vincenteli, 'Computer-aided design via optimization: A review', Automatica 18 (1982), 147-154.

[12] E.R. Panier and A.L. Tits, 'On combining feasibility, descent and superlinear convergence in inequality constrained optimization', Math. Programming 59 (1993), 261-276.

[13] R.A. Polyak, 'Smooth optimization methods for minimax problems', SIAM J. Control Optim. 26 (1988), 1274-1286.

[14] E. Polak, D.Q. Mayne and J.E. Higgins, 'A Superlinearly convergent algorithm for minimax problems', Proceedings of the 28th IEEE Conference on Decision and Control, Tampa, Florida 1 (1989), 894-898.

[15] E. Polak, D.Q. Mayne and J.E. Higgins, 'Superlinearly convergent algorithm for min-max problems', J. Optim. Theory Appl. 69 (1991), 407-439.

[16] E. Polak, D.Q. Mayne and D.M. Stimler, 'Control system design via semi-infinite optimization: A review', Proceedings of the IEEE 72 (1984), 1777-1794.

[17] M.J.D. Powell, 'A fast algorithm for nonlinearly constrained optimization calculation', in Numerical Analysis (Proc. 7th Biennial Conf., Univ. Dundee, 1977), Lecture Notes in Math. 630 (Springer-Verlag, Berlin, 1978), pp. 144-157.

[18] S.E. Salcudean, Algorithms for optimal design of feedback compensators, (Ph.D. Thesis) (Department of Electrical Engineering and Computer Sciences, University of California, Berkeley, CA, 1986).

[19] A. Vardi, 'New minimax algorithm', J. Optim. Theory Appl. 75 (1992), 613-634.

[20] T.L.S. Wuu, Delightmimo: An interactive system for optimization-based multivariable control system design, Ph.D. Thesis (Department of Electrical Engineering and Computer Sciences, University of California, Berkeley, CA, 1986).

[21] X. Yi, 'The sequential quadratic programming method for solving minimax problem', $J$. Systems Sci. Math. Sci. 22 (2002), 355-364. 
[22] Y.H. Yu and L. Gao, 'Nonmonotone line search algorithm for constrained minimax problems', J. Optim. Theory Appl. 115 (2002), 419-446.

[23] J.L. Zhou and A.L. Tits, 'Nonmonotone line search for minimax problems', J. Optim. Theory Appl. 76 (1993), 455-476.

Department of Information

Hunan Business College

410205, Changsha

Peoples Republic China

e-mail: hqj0525@126.com.cn
Institute of Applied Mathematics

Hunan University

410082, Changsha

Peoples Republic of China 